\title{
A COMMUTATIVE DIAGRAM AND AN APPLICATION TO DIFFERENTIABLE TRANSFORMATION GROUPS
}

\author{
W. D. CURTIS
}

\begin{abstract}
A commutative diagram is presented which relates the groups of concordance classes of diffeomorphisms $\Gamma\left(S^{2 n}\right)$, $\Gamma\left(C P^{n}\right)$ and $\Gamma\left(S^{2 n+1}\right)$. This diagram is applied to show that every equivariant diffeomorphism of $S^{7}$ is concordant to the identity. It follows that the exotic 8-sphere, $\Sigma^{8}$, admits no smooth semifree $S^{1}$-action with exactly two fixed points.
\end{abstract}

Introduction. In this paper we shall present a commutative diagram (Theorem 1) involving the stable homotopy group $\Pi_{1}$ and the groups $\Gamma\left(S^{n}\right), \Gamma^{+}\left(C P^{n}\right)$ defined below. This diagram is then applied to show that every orientation preserving diffeomorphism of $S^{7}$ which is equivariant with respect to the standard free action of $S^{1}$ on $S^{7}$ is concordant to the identity. Finally we show, using a result of R. Lee [3], that the exotic 8sphere, $\Sigma^{8}$, does not admit a smooth action by $S^{1}$ which is semifree with exactly two fixed points. The paper concludes with a brief discussion of possible further applications of Theorem 1 to construction of smooth actions of $S^{1}$ on exotic spheres such that the actions are semifree with exactly two fixed points.

The results of this paper are contained in the author's doctoral dissertation written at the University of Massachusetts. The author wishes to express his indebtedness to Professor J. C. Su for his generous help while the author was a graduate student. The author also acknowledges his gratitude for suggestions by a referee which resulted in a considerable shortening of the proof of Theorem 1.

Preliminaries. If $f_{0}$ and $f_{1}$ are diffeomorphisms of $M$ onto $N, M$ and $N C^{\infty}$-manifolds, we say $f_{0}$ is concordant to $f_{1}$ if there is a diffeomorphism $F: M \times I \rightarrow N \times I, I=[0,1]$, such that for all $x \in M$ we have $F(x, 0)=$ $\left(f_{0}(x), 0\right)$ and $F(x, 1)=\left(f_{1}(x), 1\right)$. The relation of concordance is an equivalence relation on the set of all diffeomorphisms of $M$ onto $N$. If $f: M \rightarrow N$ is a diffeomorphism its concordance class will be denoted [ $f$ ]. If $M$ is an orientable manifold there is a group, $\Gamma(M)$, of all concordance

Received by the editors March 15, 1971.

AMS 1970 subject classifications. Primary 55E45, 57D60, 57E25.

Key words and phrases. Homotopy sphere, stable 1-stem, Munkres-Milnor pairing, semifree circle group action, concordance, $J$-homomorphism. 
classes of orientation preserving diffeomorphisms of $M$ onto $M$. We denote by $\Gamma^{+}\left(C P^{n}\right)$ the subgroup of $\Gamma\left(C P^{n}\right)$ consisting of all $[f]$ such that $f^{*}$ preserves the generator of $H^{2}\left(C P^{n} ; Z\right)$.

The stable group $\Pi_{1}$ is equal to $\pi_{n+1}\left(S^{n}\right)$ for $n \geqq 3$. It is well known that there is an isomorphism, $J: \pi_{1}(\mathrm{SO}(n)) \rightarrow \pi_{n+1}\left(S^{n}\right)$, for $n \geqq 3$.

Main results. Before stating Theorem 1 we shall describe in some detail each of the four homomorphisms which appear in the diagram of Theorem 1.

$\gamma: \Gamma\left(S^{n}\right) \rightarrow \Gamma\left(M^{n}\right)$ : Let $M^{n}$ be a connected, oriented, smooth $n$ manifold, $\alpha \in \Gamma\left(S^{n}\right)$. We may represent $\alpha$ by a diffeomorphism $f: S^{n} \rightarrow S^{n}$ which equals the identity on a neighborhood of the lower hemisphere of $S^{n},\left\{\left(x_{0}, \cdots, x_{n}\right) \in S^{n} \mid x_{n} \leqq 0\right\}$. Let $D^{n}$ be the closed unit ball in $R^{n}$ and let $\sigma: D^{n} \rightarrow M^{n}$ be a smooth imbedding. Define $\tau:$ Int $\left(D^{n}\right) \rightarrow S^{n}$ by

$$
\tau\left(x_{1}, \cdots, x_{n}\right)=\left(x_{1}, \cdots, x_{n},\left(1-x_{1}^{2}-\cdots-x_{n}^{2}\right)^{1 / 2}\right) .
$$

Then define $\gamma(f): M^{n} \rightarrow M^{n}$ by letting $\gamma(f) \mid M^{n}-\sigma\left(D^{n}\right)$ be the identity and letting $\gamma(f) \mid \sigma\left(D^{n}\right)=\sigma\left(\tau^{-1}\right) f \tau\left(\sigma^{-1}\right)$. Since $f$ is the identity in a neighborhood of the lower hemisphere of $S^{n}$ it follows that $\gamma(f)$ is a welldefined, orientation preserving diffeomorphism. Let $\gamma(\alpha)=[\gamma(f)]$ and this is a well-defined group homomorphism.

Lemma. $\gamma: \Gamma\left(S^{2 n}\right) \rightarrow \Gamma\left(C P^{n}\right)$ takes values in $\Gamma^{+}\left(C P^{n}\right)$.

The above lemma is easily proved using Mayer-Vietoris sequences and the fact that if $W$ is a closed tubular neighborhood of $C P^{n-1}$ in $C P^{n}$ then $C P^{n}-$ Int $(W)$ is diffeomorphic to $D^{2 n}$.

$\sigma: \Gamma\left(S^{2 n}\right) \rightarrow \Gamma\left(S^{2 n}\right) \otimes \Pi_{1}: \Pi_{1} \cong Z_{2}$. Let $\sigma(\alpha)=\alpha \otimes \bar{n}$ where $\bar{n}$ is zero or nonzero depending on whether $n$ is even or odd.

$\rho: \Gamma^{+}\left(C P^{n}\right) \rightarrow \Gamma\left(S^{2 n+1}\right)$ : The circle group, $S^{1}$, acts on $S^{2 n+1}$ as follows. Let $S^{2 n+1}$ be represented as

$$
S^{2 n+1}=\left\{\left.\left(z_{0}, \cdots, z_{n}\right) \in C^{n+1}|| z_{0}\right|^{2}+\cdots+\left|z_{n}\right|^{2}=1\right\} .
$$

If $t \in S^{1}$ let $t\left(z_{0}, \cdots, z_{n}\right)=\left(t z_{0}, \cdots, t z_{n}\right)$. This action will be called the standard action of $S^{1}$ on $S^{2 n+1}$. The orbit space $S^{2 n+1} / S^{1}$ is just $C P^{n}$ and the projection $S^{2 n+1} \rightarrow C P^{n}$ is the Hopf bundle. $\Gamma^{+}\left(C P^{n}\right)$ consists of those classes, $[f]$, such that $f^{*}$ preserves the characteristic class of the above bundle. Thus $\Gamma^{+}\left(C P^{n}\right)$ consists precisely of those $[f]$ such that $f$ can be covered by an equivariant, orientation preserving diffeomorphism $f^{\prime}: S^{2 n+1} \rightarrow S^{2 n+1}$. The formula $\rho([f])=\left[f^{\prime}\right]$ is a well-defined group homomorphism.

$\rho_{2 n+1,1}: \Gamma\left(S^{2 n}\right) \otimes \Pi_{1} \rightarrow \Gamma\left(S^{2 n+1}\right):$ We assume $n \geqq 3$. This homomorphism, called the Milnor-Munkres pairing, is discussed by G. Bredon in [1]. 
Let $\quad[f] \in \Gamma\left(S^{2 n}\right), \quad[\alpha] \in\left[S^{1}, \operatorname{SO}(2 n)\right]=\Pi_{1} . \quad \rho_{2 n+1}([f] \otimes[\alpha])=[h]$ where $h: S^{2 n+1} \rightarrow S^{2 n+1}$ is defined as follows. There is a standard imbedding $S^{1} \times D^{2 n} \rightarrow S^{2 n+1}$ whose image is

$$
\left\{\left(x_{0}, \cdots, x_{2 n+2}\right) \in S^{2 n+1} \mid x_{2}^{2}+\cdots+x_{2 n+2}^{2} \leqq \frac{1}{2}\right\} .
$$

This gives a representation of $S^{2 n+1}$ as $S^{1} \times D^{2 n} \cup_{1} D^{2} \times S^{2 n-1}$ where $S^{1} \times D^{2 n}$ and $D^{2} \times S^{2 n-1}$ are identified along their common boundary, $S^{1} \times S^{2 n-1}$, by the identity map. Let $h \mid D^{2} \times S^{2 n-1}$ be the identity and let $h \mid S^{1} \times D^{2 n}$ be given by $h(z, x)=\left(z, \alpha(z) \cdot f\left(\alpha(z)^{-1} \cdot x\right)\right)$. Here we have chosen $f: S^{2 n} \rightarrow S^{2 n}$ to agree with the identity near the lower hemisphere of $S^{2 n}$. Hence we may regard $f$ as a diffeomorphism $D^{2 n} \rightarrow D^{2 n}$ which equals the identity near $S^{2 n-1}$. Thus $h$, as given above, is a diffeomorphism.

This is not the definition as given in [1] but it is not difficult to check that the two correspondences are equal.

Now our main result may be stated.

THEOREM 1. For $n \geqq 3$ the following diagram commutes.

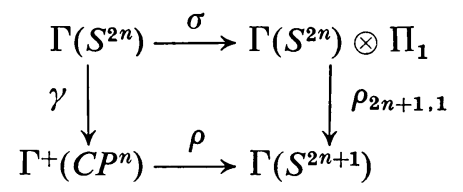

Proof. Let $[f] \in \Gamma\left(S^{2 n}\right)$. As before we may regard $f$ as a diffeomorphism $f: D^{2 n} \rightarrow D^{2 n}$ which equals the identity in a neighborhood of $S^{2 n-1}$. Represent $C P^{n}$ as $D^{2 n} \cup_{1} W$ where $W$ is the total space of the 2-disk bundle associated to the Hopf bundle $S^{2 n-1} \rightarrow C P^{n-1}$. There is a natural action of $S^{1}$ on $D^{2} \times S^{2 n-1}$ by $t(x, y)=(t x, t y)$ where $t x$ is complex multiplication and $t y$ is the action of $t$ on $y$ with respect to the standard action. Now $W=\left(D^{2} \times S^{2 n-1}\right) / S^{1}$ so there is a projection $D^{2} \times S^{2 n-1} \rightarrow$ $W$. Also $S^{1}$ acts on $S^{1} \times D^{2 n}$ by $t(z, x)=(t z, t x)$. The orbit manifold is $D^{2 n}$. The identity map

$$
\partial\left(S^{1} \times D^{2 n}\right) \rightarrow \partial\left(D^{2} \times S^{2 n-1}\right)
$$

is equivariant so there is a smooth $S^{1}$-action on $S^{1} \times D^{2 n} \cup_{1} D^{2} \times S^{2 n-1}$. The principal $S^{1}$-bundle

$$
S^{1} \times D^{2 n} \cup_{1} D^{2} \times S^{2 n-1} \rightarrow D^{2 n} \cup_{1} W
$$

is equivalent to the Hopf bundle. Let $\theta: S^{1} \rightarrow U(n) \subset \mathrm{SO}(2 n)$ be given by $\theta(z)=z I$. Define $g: S^{1} \times D^{2 n} \cup_{1} D^{2} \times S^{2 n-1} \rightarrow S^{1} \times D^{2 n} \cup_{1} D^{2} \times S^{2 n-1}$ by $g \mid D^{2} \times S^{2 n-1}=$ identity and, for $(z, x) \in S^{1} \times D^{2 n}, g(z, x)=$ $\left(z, \theta(z) \cdot f\left(\theta(z)^{-1} x\right)\right) \cdot g$ is an equivariant diffeomorphism covering $f \cup 1$ Thus $\rho \gamma([f])=[g]$.

Now $[\theta] \in \Pi_{1}=\left[S^{1}, \operatorname{SO}(2 n)\right]$. It is known [1] that if $\psi: S^{1} \rightarrow \operatorname{SO}(2 n)$ 
is defined by

where

$$
\psi\left(e^{i t}\right)=\left[\begin{array}{cccc}
A^{r_{1}} & 0 & \cdots & 0 \\
0 & A^{r_{2}} & & \\
\cdot & & \cdot & \\
\cdot & & \cdot & \\
\cdot & & \cdot & \\
0 & & & A^{r_{n}}
\end{array}\right]
$$

$$
A=\left[\begin{array}{rr}
\cos t & -\sin t \\
\sin t & \cos t
\end{array}\right],
$$

then in $\Pi_{1}, \psi \equiv r_{1}+\cdots+r_{n}(\bmod 2)$, i.e., $\psi$ is zero or nonzero depending on whether $r_{1}+\cdots+r_{n}$ is even or odd. $\theta$ is of the form $\psi$ with all $r_{i}=1$. So $\theta$ is zero or nonzero depending on whether $n$ is even or odd. Looking at the definition of $\rho_{2 n+1,1}$ we see $\rho_{2 n+1,1}(\sigma([f]))=[g]=\rho \gamma([f])$ as asserted. This proves the theorem.

Applications of Theorem 1. We are not able to give many applications of Theorem 1 due to a general lack of knowledge of the maps involved. However, in case $n=3$, we have some results of G. Bredon [1] and R. Lee [3]. Specifically,

TheOREM (R. LeE). $\quad \gamma: \Gamma\left(S^{6}\right) \rightarrow \Gamma\left(C P^{3}\right)$ is onto.

THEOREM (G. BREDON). $\rho_{7,1}: \Gamma\left(S^{6}\right) \otimes \Pi_{1} \rightarrow \Gamma\left(S^{7}\right)$ is trivial.

In case $n=3$ the diagram of Theorem 1 becomes

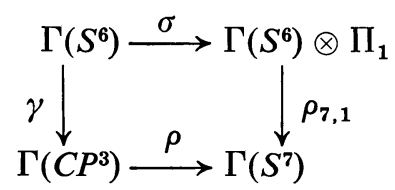

Since $\rho_{7,1}$ is trivial and $\gamma$ is onto we have:

THEOREM 2. $\rho: \Gamma\left(C P^{3}\right) \rightarrow \Gamma\left(S^{7}\right)$ is trivial, i.e., every orientation preserving diffeomorphism of $S^{7}$ which is equivariant with respect to the standard action of $S^{1}$ on $S^{7}$ is concordant to the identity.

REMARK. By [2], $\Gamma\left(S^{7}\right) \cong Z_{2}$ so there do exist orientation preserving diffeomorphisms of $S^{7}$ which are not concordant to the identity. Of course by Theorem 2 no such map can be equivariant.

Using a classification theorem of $R$. Lee [3] we now give an application of Theorem 2 to transformation groups.

If a group $G$ acts on a set $X$ the action is called semifree if whenever $g \in G$ and $x \in X$ are such that $g x=x$ then either $x$ is a fixed point or $g$ 
is the identity of $G$. In other words, if $F$ is the set of fixed points of the action then the action of $G$ on $X-F$ is free.

Let $S^{1}$ act on $D^{2 n}$ by $t\left(z_{1}, \cdots, z_{n}\right)=\left(t z_{1}, \cdots, t z_{n}\right)$ where

$$
D^{2 n}=\left\{\left.\left(z_{1}, \cdots, z_{n}\right) \in C^{n}\left|\sum_{i=1}^{n}\right| z_{1}\right|^{2} \leqq 1\right\} .
$$

The restriction of this action to $S^{2 n-1}$ is the standard action. Suppose $f: S^{2 n-1} \rightarrow S^{2 n-1}$ is an orientation preserving equivariant diffeomorphism. Then $D^{2 n} \cup_{f} D^{2 n}$ is a homotopy $2 n$-sphere and it carries a smooth semifree action with exactly two fixed points. R. Lee [3] has shown that if $\left(S^{1}, \Sigma^{2 n}\right)$ is any semifree $S^{1}$-action on the homotopy sphere $\Sigma^{2 n}$ with exactly two fixed points then there is an $f$ as above such that $\left(S^{1}, \Sigma^{2 n}\right)$ is equivalent to the action of $S^{1}$ on $D^{2 n} \cup_{f} D^{2 n}$ constructed above. Using this we prove:

TheOREM 3. Let $\Sigma^{8}$ be the exotic 8-sphere. Then $\Sigma^{8}$ does not admit a smooth, semifree $S^{1}$-action with exactly two fixed points.

Proof. According to Lee's result, if such an action did exist then $\Sigma^{8}$ would be expressible as $D^{8} \cup_{f} D^{8}$ where $f: S^{7} \rightarrow S^{7}$ is an equivariant, orientation preserving diffeomorphism. But by Theorem 2 such an $f$ is concordant to the identity. Hence $\Sigma^{8}$ would be diffeomorphic to $S^{8}$ contrary to assumption.

In closing we make a remark concerning the possibility of further applications of Theorem 1 to transformation groups. Suppose for some odd $n$ we knew $\rho_{2 n+1,1}$ was nontrivial. Since $\sigma: \Gamma\left(S^{2 n}\right) \rightarrow \Gamma\left(S^{2 n}\right) \otimes \Pi_{1}$ is onto for $n$ odd we could conclude that $\rho: \Gamma\left(C P^{n}\right) \rightarrow \Gamma\left(S^{2 n+1}\right)$ is nontrivial. Therefore we would have an orientation preserving, equivariant diffeomorphism $f: S^{2 n+1} \rightarrow S^{2 n+1}$ which is not concordant to the identity. Thus $D^{2 n+2} \cup \cup_{f} D^{2 n+2}$ is an exotic homotopy sphere. Thus we would have constructed a smooth, semifree action with exactly two fixed points of $S^{1}$ on an exotic sphere $\Sigma^{2 n+2}$. However the author is unaware of any example of an odd $n$ where $\rho_{2 n+1,1}$ is nontrivial.

\section{REFERENCES}

1. G. Bredon, $A \Pi_{*}$-module structure for $\Theta_{*}$ and applications to transformation groups, Ann. of Math. (2) 86 (1967), 434-448. MR 36 \#4570.

2. M. Kervaire and J. Milnor, Groups of homotopy spheres. I, Ann. of Math. (2) 77 (1963), 504-537. MR 26 \#5584.

3. R. Lee, Differentiable classification of some topologically linear actions, Dissertation, University of Michigan, 1968.

Department of Mathematics, Kansas State University, Manhattan, KANSAS 66502 